Pacific Journal of Mathematics

LOCAL RINGS WITH NOETHERIAN FILTRATION 


\title{
LOCAL RINGS WITH NOETHERIAN FILTRATIONS
}

\section{CHIN-PI LU}

\begin{abstract}
The purpose of this paper is to investigate a certain type of complete filtered local rings, and to generalize well-known theorems in literature which are related to the structure theorem of complete local rings.
\end{abstract}

All rings in this article are commutative with identity element, and all subrings possess the identity element of the containing ring. A ring $A$ is a local ring if it has a unique maximal ideal $m$, symbolically we write $(A, m)$, and by the natural topology of $(A, m)$ we mean the $m$-adic topology.

A filtration $\left\{q_{n}\right\}_{n=0}^{\infty}$ of a ring $R$ is called a noetherian filtration if it is separated and $R / q_{n}$ is a noetherian ring for all $n$. Motivated by I. S. Cohen's structure theorem of complete noetherian local rings with respect to the natural topologies ([5]), extension of two theorems on noetherian local rings is considered. Harrison-Kolman's theorem [6, Th. A, Th. 1], which is annalogous to Cohen's theorem, is generalized as follows: If $R$ is a local ring which is complete with respect to a noetherian filtration $\left\{q_{n}\right\}_{n=0}^{\infty}$ such that (i) $R$ is equicharacteristic and (ii) $R / q_{1}$ is regular, then there exists a subring $B$ such that $B+q_{1}=R$ and $B \cap q_{1}=(0)$; consequently, $R$ is a homomorphic image of a ring of formal power series in a finite or countably infinite number of indeterminates over $B$. We also prove that if $R$ is a complete (resp. compact) local ring with respect to a noetherian filtration $\left\{q_{n}\right\}_{n=0}^{\infty}$ such that $q_{1}$ is its maximal ideal, then $R$ is a homomorphic image of a ring $k\left[\left[x_{i}\right]\right]_{i \in I}$ of formal power series in a finite or countably infinite number of indeterminates over a complete (resp. compact) noetherian local subring $k$ for the natural topology; conversely, such power series ring possesses a noetherian filtration and $k\left[\left[x_{i}\right]\right]_{i \in I} / D$, for any closed ideal $D$ of $k\left[\left[x_{i}\right]\right]_{i \in I}$ relative to the filtration topology, is a complete (resp. compact) local ring with respect to a noetherian filtration. This is an extension of Cohen-Warner's theorem ([5, Th. 9, 12]; [11, Th. 14]). Finally, we apply the result to prove that a certain class of local rings are unique factorization domains.

1. Natural topologies of power series rings. Let $N$ be the set of all positive integers, and $N_{0}=N \cup\{0\}$. Throughout the paper, we shall denote the power series ring $R\left[\left[x_{1}, x_{2}, \cdots, x_{t}\right]\right]$ in $t$ indeterminates over a ring $R$ by $R_{t}$ for each $t \in N_{0}$, identifying $R_{0}$ with $R$. Also the power series ring $R\left[\left[x_{1}, x_{2}, \cdots\right]\right]=R\left[\left[x_{i}\right]\right]_{i \in N}$ in a countably 
infinite number of indeterminates over $R$ will be denoted by $R_{\omega}$. Let $f=f\left(x_{1}, x_{2}, x_{3}, \cdots\right)$ be an element of $R_{\omega}$, then the power series $f\left(x_{1}, x_{2}, \cdots, x_{t}, 0,0,0, \cdots\right)$ in $R_{t}$ is called the projection of $f$ on $R_{t}$ and is denoted by $(f)_{t}$. For any $t \in N_{0}$ the projection map $P_{t}: f \rightarrow(f)_{t}$ is a ring homomorphism of $R_{\omega}$ onto $R_{t}$.

Let $R$ be a ring with a filtration, that is a descending sequence of ideals $R=q_{0} \supseteqq q_{1} \supseteqq \cdots \supseteqq q_{n} \supseteqq \cdots$ such that $q_{i} q_{j} \supseteqq q_{i+j}$. If $\bigcap_{n \in N_{0}} q_{n}=(0)$, then $R$ is separated for the topology induced by the filtration; furthermore, we can define an order function $v$ on $R$ such that

$$
\begin{array}{rrr}
v(a) & =\min \left\{n \mid a \notin q_{n+1}\right\} & \text { if } 0 \neq a \in R \\
& =\infty & \text { if and only if } a=0 .
\end{array}
$$

The sets $R_{t}, t \in N_{0}$, and $R_{\omega}$ of formal power series over $R$ are the sets $R^{N^{t}}$ and $R^{N^{(N)}}$ respectively. The (cartesian) product topology of $R_{t}$ and that of $R_{\omega}$ are compatible with the ring structure of the power series rings. We shall call it the natural topology induced by the filtration $\left\{q_{n}\right\}$ of $R$, or the $\left(q_{n}\right)$-natural topology. If $q_{n}=m^{n}$, $n \in N_{0}$, for some ideal $m$ of $R$, then the induced natural topology on a power series ring is simply called the m-natural topology.

Let $\left\{n_{i}\right\}$ be any sequence of positive integers $n_{i}$ such that $n_{i} \rightarrow \infty$ as $i \rightarrow \infty$, and $A_{r}=\left\{f \in R_{\omega} \mid\right.$ each nonzero monomial $b x_{1}^{\lambda_{1}} x_{2}^{\lambda_{2}} \cdots x_{t}^{\lambda_{t}}$ of $f$ satisfies that $\left.v(b)+\sum_{1 \leqq i \leqq t} n_{i} \lambda_{i} \geqq r\right\}$ for each $r \in N_{0}$. Then $\left\{A_{r}\right\}_{r \in N_{0}}$ forms a filtration of $R_{\omega}$ and therefore induces a linear topology on $R_{\omega}$. We note that the linear topology is independent of the choice of the sequence $\left\{n_{i}\right\}$ of positive integers $n_{i}$ such that $n_{i} \rightarrow \infty$ as $i \rightarrow \infty$. In particular, we take the sequence $\left\{n_{i}\right\}$ such that $n_{i}=i$ for every $i$, and for each $r \in N_{0}$ let $B_{r}=\left\{f \in R_{\omega} \mid\right.$ each nonzero monomial $b x_{1}^{\lambda_{1}} x_{2}^{\lambda_{2}} \cdots x_{t}^{\lambda_{t}}$ of $f$ satisfies that $\left.v(b)+\sum_{i \leqq i \leqq t} i \lambda_{i} \geqq r\right\} . \cdots\left(^{*}\right)$. Then it is not difficult to verify that the natural topology of $R_{\omega}$ induced by the filtration of $R$ is equivalent to the $\left(B_{r}\right)$-topology; hence to the $\left(A_{r}\right)$-topology for any sequence $\left\{n_{i}\right\}$ of positive integers $n_{i}$ tending to $\infty$. Consequently, the $\left(q_{n}\right)$-natural topology of each $R_{t}$ could be defined by the basis of neighborhoods $\left\{A_{t, r}\right\}$ of 0 , where $A_{t, r}=R_{t} \cap A_{r}$ for each $r \in N_{0}$. If $m$ is an ideal of $R$ such that $\bigcap_{n \in N_{0}} m^{n}=(0)$, then the $m$-natural topology of $R_{t}$ is equivalent to the $\left(m, x_{1}, x_{2}, \cdots, x_{t}\right)$-adic topology, because the latter is obviously the $\left(A_{t, r}\right)$-topology with respect to any sequence $\left\{n_{i}\right\}$ of positive integers $n_{i}$ tending to $\infty$ such that $n_{1}=n_{2}=\cdots=n_{t}=1$. In addition, if $(R, m)$ is a local ring, then the $m$-natural topology of $R_{t}$ is identical with the natural topology of the local ring $\left(R_{t}, M\right)$, where $M=\left(m, x_{1}, x_{2}, \cdots, x_{t}\right)$.

Since each $R_{t}$ can be canonically identified with a subset $\prod_{\alpha \in N^{(N)}} S_{\alpha}$ 
of $R_{\omega}$, wehre $S_{\alpha}=R$ for some $\alpha$ 's and $S_{\alpha}=(0)$ for all other $\alpha$, the following proposition is trivially true.

Proposition 1. If $R$ is a ring with a separated filtration $\left\{q_{n}\right\}$, then every $R_{t}, t \in N_{0}$, is closed in $R_{\omega}$ which is equipped with the $\left(q_{n}\right)$-natural topology.

THEOREM 1. Let $R$ be a ring with a separated filtration $\left\{q_{n}\right\}$, and let $R_{\omega}$ and each $R_{t}$ be equipped with the $\left(q_{n}\right)$-natural topologies respectively. Then the following statements are equivalent: (1) $R$ is compact [a Hausdorff space, complete, linearly compact (cf. [4]), precompact]. (2) $R_{\omega}$ is compact [the same nature as (1)]. (3) $R_{t}$ is compact [the same nature as (1)] for all $t \geqq 1$.

Proof. The theorem follows from Proposition 1 and well known properties of products of topological rings (cf. [2], [3], [4]).

2. Local rings with noetherian filtrations. In this section we assume that every filtration $\left\{q_{n}\right\}$ of rings $R$ is separated.

Let $\operatorname{gr}(R)$ be the associated graded ring of a filtered ring $R$ with respect to a filtration $\left\{q_{n}\right\}, k$ a subring of $R, \rho$ the natural homomorphism of $R$ to $\operatorname{gr}_{0}(R)=q_{0} / q_{1}$ and $\sigma$ the restriction of $\rho$ to $k$. Then $\operatorname{gr}(R)$ has a structure of $k$-algebra if the product $c \cdot x$ for each $c \in k$ and $x \in \operatorname{gr}(R)$ is defined as $\sigma(c) x$. Given $0 \neq a \in R$, the class of $a$ in $\operatorname{gr}_{i}(R)$ where $i=v(a)$ is called the initial form of $a$ and is denoted by $a^{*}$.

Proposition 2. Let $R$ be a ring with a filtration $\left\{q_{n}\right\}, k$ its subring and $I=\{1,2, \cdots, j\}$ for some $j \in N($ resp. $I=N)$. Let $S=\left\{a_{i}\right\}_{i \in I}$ be a set of elements of $R$ such that $v\left(a_{i}\right)=n_{i}>0$ for each $i$ (resp. $v\left(a_{i}\right)=n_{i}>0$ and $n_{i} \rightarrow \infty$ as $\left.i \rightarrow \infty\right)$. (1) If $R$ is complete and $S \cap k$ has at most one element, then there exists a unique k-homomorphism $u$ of the k-algebra $k_{j}\left(\right.$ resp. $\left.k_{\omega}\right)$ into $R$ such that $u\left(x_{i}\right)=a_{i}$ for every $i \in I$, which is continuous for the $\left(k \cap q_{n}\right)$-natural topology of $k_{j}\left(\operatorname{resp} . k_{\omega}\right)$. (2) If the family $\left\{a_{i}^{*}\right\}_{i \in I}$ of the initial forms of $a_{i}$ 's is a system of generators of the k-algebra $\operatorname{gr}(R)$, then the homomorphism $u$ in (1) is suvjective.

Proof. First we assume that $I=N$. For the sequence of positive integers $\left\{n_{i}\right\}$ and the filtration $\left\{q_{n}^{\prime}\right\}$ of $k$, where $q_{n}^{\prime}=(0)$ for every $n$, let $A_{r}$ for each $r \in N_{0}$ be the ideal of $k_{\omega}$ which was defined in $\S 1$. Then $k_{\omega}$ is separated and complete for the $\left(A_{r}\right)$-topology, namely, the (0)-natural topology by Theorem 1 and has $\bar{k}=U_{t \in N} k\left[x_{1}, x_{2}, \cdots, x_{t}\right]$ 
as a dense subset. Regarding $\bar{k}$ as a subspace of $k_{\omega}$, let $\eta$ be the $k$ homomorphism of the $k$-algebra $\bar{k}$ to $R$ such that $\eta\left(x_{i}\right)=a_{i}$ for every $i$, then $\eta$ is a continuous ring homomorphism and can be extended uniquely to a continuous homomorphism $u$ of $k_{\omega}$ to $R$ with respect to the (0)-natural topology of $k_{\omega}$. The proofs of this and (2) are completely analogous to that of Proposition 11 of $[4$, p. 40]; hence we omit them. Note that if $S \cap k=\left\{a_{1}\right\}$, then $u: k_{\omega} \rightarrow R$ is actually a homomorphism of $k\left[\left[x_{2}, x_{3}, x_{4}, \cdots\right]\right]$ to $R$. The continuity of $u$ for the $\left(k \cap q_{n}\right)$-natural topology of $k_{\omega}$ can be proved very easily by applying the following fact: If $v(b)+\sum_{1 \leqq i \leqq t} n_{i} \lambda_{i} \geqq r$, then

and

$$
u\left(b x_{1}^{\lambda_{1}} x_{2}^{\lambda_{2}} \cdots x_{t}^{\lambda^{t}}\right)=b a_{1}^{\lambda_{1}} a_{2}^{\lambda_{2}} \cdots a_{t}^{\lambda_{t}}
$$

$$
v\left(b a_{1}^{\lambda_{1}} a_{2}^{\lambda_{2}} \cdots a_{t}^{\lambda_{t}}\right) \geqq v(b)+\sum_{1 \leqq i \leqq t} n_{i} \lambda_{i} \geqq r .
$$

When $I=\{1,2, \cdots, j\}$ the proof of the proposition is similar to that of the above case.

Definition 1. A filtration $\left\{q_{n}\right\}_{n \in N_{0}}$ of a ring $R$ is called a noetherian filtration if $\bigcap_{n \in N_{0}} q_{n}=(0)$ and $R / q_{n}$ is a noetherian ring for all $n$.

If $R$ is a noether ring with a filtration $\left\{q_{n}\right\}$, then the induced filtration $\left\{B_{n}\right\}$ on $R_{\omega}$, where $B_{n}$ is defined by $\left(^{*}\right)$ in $\S 1$, is a noetherian filtration. For if we let $P_{n}, n \in N_{0}$, be the projection map of $R_{\omega}$ to $R_{n}$ such that $P_{n}(f)=(f)_{n}$ for each $f \in R_{\omega}$ and $\eta_{n}$ the natural homomorphism of $R_{n}$ to $R_{n} /\left(R_{n} \cap B_{n+1}\right)$, then $\operatorname{ker}\left(\eta_{n} \circ P_{n}\right)=B_{n+1}$; therefore $R_{\omega} / B_{n+1} \cong R_{n} /\left(R_{n} \cap B_{n+1}\right)$, which is a noetherian ring for every $n$. Moreover $\bigcap_{n \in N_{0}} B_{n}=(0)$ by Theorem 1 .

DEFINITION 2. A set $\left\{a_{i}\right\}_{i \in I}$ of elements of a filtered ring $R$ is called a formal system of generators for $R$, if the set $\left\{a_{i}^{*}\right\}_{i \in I}$ of the initial forms of $a_{i}$ 's forms a minimal basis for the homogeneous ideal $\bigoplus_{i \geqq 1} \operatorname{gr}_{i}(R)$ of $\operatorname{gr}(R)$.

Let $\left\{a_{\imath}\right\}_{i \in I}$ be a formal system of generators for a ring $R$ with a filtration $\left\{q_{n}\right\}$. According to Proposition 1 of $[4$, p. 9], the set $\left\{a_{i}^{*}\right\}_{i \in I}$ is also a minimal set of generators for the $\operatorname{gr}_{0}(R)$-algebra $\operatorname{gr}(R)$. If $k$ is a subring of $R$ such that $k+q_{1}=R$, then the above conclusion is also true for $\operatorname{gr}(R)$ as a $k$-algebra.

We remark that if one formal system of generators for $R$ is of the finite type (resp. countably infinite type), then so are all other formal systems of generators by virtue of the minimal condition imposed on the set of the initial forms of generators. 
The following proposition is easy to see.

Proposition 3. Let $R$ be a ring with a noetherian filtration. Then (1) $R$ satisfies the ascending chain condition for open ideals, (2) a homomorphic image and the completion of $R$ possess noetherian filtrations respectively, and (3) if $R$ is complete and possesses a formal system of generators of the finite type, then $R$ is noetherian (cf. [4, p. 42, Corollary 2 to Proposition 12]; [4, p. 45, Corollary 4 to Theorem 2]).

LEMMA. Every ring $R$ with a noetherian filtration $\left\{q_{n}\right\}$ possesses a formal system of generators, and each formal system $\left\{a_{i}\right\}_{i \in I}$ consists of a countable (finite or infinite) number of elements such that $v\left(a_{i}\right) \rightarrow \infty$ as $i \rightarrow \infty$ when $I=N$.

Proof. The first part is evident from [12, p. 151, Th. 7]. Since each finite basis for the ideal $g r_{i}(R)$ of the noetherian ring $R / q_{i+1}$ is also a set of generators for $\operatorname{gr}_{i}(R)$ as a $\operatorname{gr}_{0}(R)$-module, $\bigoplus_{i \geqq 1} \operatorname{gr}_{i}(R)$ is a module generated by a countable number of homogeneous elements over $\operatorname{gr}_{0}(R)$; hence over $\operatorname{gr}(R)$. This yields that all other formal systems of generators for $R$ must be countable as we remarked previously. To verify the remaining part of the lemma, assume the contrary and let $S=\left\{a_{i}\right\}_{i \in N}$ be a formal system of generators such that $v\left(a_{i}\right) \neq \infty$ as $i \rightarrow \infty$, then there exists a $t \in N_{0}$ and an infinite subset $\left\{a_{j_{i}} \in S \mid i \in N\right.$ and $\left.v\left(a_{j_{i}}\right)=t\right\}$ of $S$. Since $\left\{a_{i}^{*}\right\}_{i \in N}$ is a minimal basis for the ideal $\bigoplus_{i \geqq 1} \mathrm{gr}_{i}(R)$, the chain of ideals

$$
\left(a_{j_{1}}^{*}\right) \subset\left(a_{j_{1}}^{*}, a_{j_{2}}^{*}\right) \subset\left(a_{j_{1}}^{*}, a_{j_{2}}^{*}, a_{j_{3}}^{*}\right) \subset \cdots
$$

in the noetherian ring $R / q_{t+1}$ does not terminate, forming a contradiction.

Definition 3. A local ring $R$ with a filtration $\left\{q_{n}\right\}$ is called $a$ special local ring with respect to the $\left(q_{n}\right)$-topology (or the filtration $\left.\left\{q_{n}\right\}\right)$ if it satisfies the following properties: (1) $R$ is equicharacteristic, (2) $R / q_{1}$ is a regular noetherian local ring, (3) $R$ is complete for the $\left(q_{n}\right)$-topology.

The combination of Harrison's and Kolman's theorems in [6] (Theorem A and Theorem 1) can be stated as follows:

THEOREM $\alpha$. Let $R$ be a special noetherian local ring with respect to the A-adic Hausdorff topology for some ideal $A$ of $R$, then there exists a subring $B$ of $R$ such that $B+A=R$ and $B \cap A=(0)$. 
Now we consider an extension of this theorem.

THEOREM 2. Let $R$ be a special local ring with respect to a noetherian filtration $\left\{q_{n}\right\}$, then there exists a subring $B$ of $R$ such that $B+q_{1}=R$ and $B \cap q_{1}=(0)$.

Proof. If $q_{2}=(0)$, then $q_{1}^{2}=(0)$ and $R / q_{2} \cong R$. Hence, $R$ is a special noetherian local ring for the $q_{1}$-adic topology which is discrete, and the theorem is identical with Theorem $\alpha$. For the case $q_{2} \neq(0)$, the proof is similar to that of Theorem 1 of [6], in which we only need to replace each $A^{i}$ with $q_{i}$ and the $A$-adic topology with the $\left(q_{n}\right)$-topology.

COROLlaRy. Let $R$ be a special local ring with respect to a noetherian filtration $\left\{q_{n}\right\}$. Then $R$, as a topological ring, is a homomorphic image of a power series ring in a finite or countably infinite number of indeterminates over a subring $B$ of $R$ such that $B+q_{1}=R$ and $B \cap q_{1}=(0)$, where the power series ring is equipped with the $\left(B \cap q_{n}\right)$-natural topology.

Proof. The corollary can be proved by successive applications of Theorem 2, Lemma, the remark following Definition 2 and Proposition 2.

Generalizing I. S. Cohen's structure theorem of complete noetherian local rings for the natural topologies ([5]), Nagata proved that embedding of coefficient rings in separated complete nonnetherian local rings for the natural topologies is also possible ([8]). A similar attempt to extend the embedding theorem to a wider class of complete local rings was made by Roquette ([10]). He proved that a complete local ring $(R, M)$ equipped with a Hausdorff linear topology, which is weaker than the natural topology, possesses a complete local subring $(k, m)$ such that $R=k+M . \quad k$ is either a field or a $v$-ring according as $R$ is equicharacteristic or not, and in the latter case the natural topology of $k$ is equivalent to the topology induced on $k$ as a subset of $R$ ([10, p. 76, Satz 1]; [10, p. 86, §1.4.(b)]). Applying Roquette's theorem and our results so far, we can extend Theorem 9 and Theorem 12, both of [5], and Theorem 14 of [11] as follows.

TheOREM 3. Let $(R, M)$ be a complete (resp. compact) local ring with respect to a noetherian filtration $\left\{q_{n}\right\}$ such that $q_{1}=M$. Then (1) $R$, as a topological ring, is a homomorphic image of a power series ring $k\left[\left[x_{i}\right]\right]_{i \in I}$ in a finite or countably infinite number of indeterminates over a complete (resp. compact) local ring $(k, m)$ for the natural topology; where $k$ is either $R / M$ (resp. the finite field $R / M$ ) 
or a v-ring whose residue field is $R / M$ according as $R$ is equicharacteristic or not, and the power series ring is equipped with the $m$ natural topology. (2) Conversely, if $D$ is any closed ideal of such power series ring, then $k\left[\left[x_{i}\right]\right]_{i \in I} / D$ is a complete (resp. compact) local ring with respect to a noetherian filtration.

Proof. Since $q_{1}=M$, the $\left(q_{n}\right)$-topology of $R$ is weaker than the natural topology; hence there exists a coefficient ring $(k, m)$ which is either a field or a $v$-ring such that $m=(p)$ and $k / m \cong R / M$ due to Roquette's theorem, where $p$ is the characteristic of $R / M$. According to Lemma, $R$ possesses a formal system of generators, say, $S=\left\{a_{i}\right\}_{i \in I}$ for some $I \subseteq N$. If $k$ is a field, then $S \cap k=\varnothing$ as $v\left(a_{i}\right)>0$ for all $i$. If $k$ is a $v$-ring, then $S \cap k$ has at most one element. To see this, let $a_{i}$ and $a_{j}$ be two distinct elements of $S \cap k$. Then $a_{i}, a_{j} \in m$, so that $a_{i}=p^{e} \alpha$ and $a_{j}=p^{\lambda} \beta$ for some $e, \lambda \in N$ and some units $\alpha$ and $\beta$ of $k$. Assume that $e \geqq \lambda$, then $a_{i}=p^{e} \alpha=\left(p^{\lambda} \beta\right)\left(p^{e-i} \alpha \beta^{-1}\right)=$ $a_{j} y$, where $y=p^{e-\lambda} \alpha \beta^{-1} \in k$; consequently, $a_{i}^{*}=a_{j}^{*} y^{*}$, which is a contradiction because $\left\{a_{i}^{*}\right\}_{i \in I}$ is a minimal basis for $\bigoplus_{i \geqq 1} g_{i}(R)$. Now the theorem can be proved by the same method as in the proof of corollary to Theorem 2. Note that the $\left(k \cap q_{n}\right)$-natural topology of $k\left[\left[x_{i}\right]\right]_{i \in I}$ and the $m$-natural topology are equivalent since the $\left(k \cap q_{n}\right)$ topology and the natural topology of $(k, m)$ are equivalent. When $R$ is compact, the compactness or the finiteness of $k$ follows from Lemma 3 and Theorem 7, both of [11]. (2) is trivially true for the case that $I$ is finite. If $I=N$, then $k\left[\left[x_{i}\right]\right]_{i \in I}=k_{\omega}$ and $\left\{\left(B_{n}+D\right) / D\right\}_{n \in N_{0}}$ is a noetherian filtration of the local ring $k_{\omega} / D$ due to the remark following Definition 1. The completeness or the compactness of $k_{\omega} / D$ with respect to the noetherian filtration can be proved by applying Theorem 1 and the fact that $k_{\omega}$ is a Hausdorff metric space for the $m$-natural topology (cf. [3, p. 40, p. 48]).

CoROllaRY 1. A strictly linearly compact local ring $(R, M)$, with respect to a filtration $\left\{q_{n}\right\}_{n \in N_{0}}$ such that $q_{1}=M$, is a homomorphic image of a formal power series ring in a finite or a countably infinite number of indeterminates over a strictly linearly compact subring $k$ of $R$ which is either field or a v-ring.

Proof. Evidently $\left\{q_{n}\right\}$ is a noetherian filtration because $R / q_{n}$ is an artinian ring for each $n$; hence the corollary follows immediately from Theorem 3 (cf. [4, p. 110, excer. 19, excer. 22, (b)]).

The above corollary is identical with Theorem 1 of [1] relative to our particular linear topology. 
CoROllaRY 2. Let $(R, M)$ be a complete local ring with respect to a noetherian filtration $\left\{q_{n}\right\}$ such that $q_{1}=M$. If $R$ possesses a formal system $S=\left\{a_{i}\right\}_{i \in I}$ of generators of the finite type, then it is a complete noetherian local ring for the natural topology, and $M$ is generated by $S$. Consequently, the $\left(q_{n}\right)$-topology is equivalent to the natural topology.

Proof. Assume that $I=\{1,2, \cdots, j\}$, and let $u$ be the homomorphism of $k_{j}$ onto $R$ stated in (1) of Theorem 3; where $k$ is either a field or a $v$-ring with the maximal ideal $m$. Then evidently $k_{j}$ is a complete noetherian local ring for the natural topology. According to [4, p. 41, Proposition 12], $M$ is generated by $S$; hence it is the image of the maximal ideal of $k_{j}$ under the $k$-homomorphism $u$. Consequently, $u$ is continuous for the natural topology of $k_{j}$ and that of $R$, and $R=u\left(k_{j}\right)$ is a noetherian local ring which is complete for the natural topology. It follows that the $\left(q_{n}\right)$-topology and the natural topology of $R$ are equivalent by [12, p. 270, Th. 13].

The next theorem is similar to [5, p. 88, Th. 15].

THEOREM 4. Let $(R, M)$ be a complete local ring with respect to a noetherian filtration $\left\{q_{n}\right\}$ such that $q_{1}=M, S=\left\{a_{i}\right\}_{i \in I}$ a formal system of generators for $R$, and $p$ the characteristic of $R / M$. Suppose that $R$ is equicharacteristic (resp. $R$ is unequicharacteristic and $p \in S)$. If elements of each finite subset of $S$ are analytically independent, then as a topological ring, $R$ is isomorphic to a power series ring $k\left[\left[x_{i}\right]\right]_{i \in I}$ (resp. $k\left[\left[x_{i}\right]\right]_{i \in I-\{1\}}$ ) in a finite or countably infinite number of indeterminates over a field $k$ (resp. a v-ring $(k, m)$ whose residue field is $R / M)$, where the power series ring is equipped with the (0)-natural topology (resp. the m-natural topology). Consequently, $R$ is a unique factorization domain. (2) Conversely, such power series ring is a complete local ring with respect to a noetherian filtration having $T=\left\{x_{i}\right\}_{i \in I}$ (resp. $T=\{p\} U\left\{x_{i}\right\}_{i \in I}$ ) as a formal system of generators, and $T$ satisfies the condition that elements of each finite subset are analytically independent.

Proof. Let us put $p=a_{1}$ if $p \in S$. Assume that $I$ is finite. Then $R$ is a complete noetherian local ring for the natural topology, and $M$ is generated by $S$ due to Corollary 2 to Theorem 3. Moreover, $S$ is a minimal basis for $M$ and $R$ is regular, because the elements of $S$ are analytically independent (cf. [9, p. 72, Th. 4]). If $R$ is unequicharacteristic and $p \in S$, then $p \notin M^{2}$ by $[9$, p. 69, Proposition 6]; thus $R$ is an unramified complete regular noetherian local ring. Now the theorem is well known by $[5$, p. 88 , Th. 15]). Note that 
the (0)-natural topology (resp. the m-natural topology) of the power series ring, which is a local ring, is equivalent to its natural topology due to a remark in $\S 1$. Next, we assume that $I=N$. We shall prove that the $k$-homomorphism $u$ of $k_{\omega}$ to $R$ considered in (1) of Theorem 3 is an isomorphism; we do this only for the case that $k$ is a $v$-ring, since the proof for the other case is similar. Because $p=a_{1} \in S$, we may consider $u$ as a $k$-homomorphism such that $u\left(x_{i}\right)=a_{i+1}$ for all $i \geqq 1$. Put $k^{\prime}=U_{i \in N} k_{i}, u^{\prime}=u \mid k^{\prime}$ and $u_{i}=u \mid k_{i}$ for each $i$, then each $u_{i}\left(k_{i}\right)=R^{(i)}$ is a noetherian local ring with the maximal ideal $m^{(i)}$ generated by $S_{i}=\left\{a_{1}, a_{2}, \cdots, a_{i+1}\right\} \subset M$; hence $m^{(i)}=M \cap R^{(i)}$ and the elements of $S_{i}$ are analytically independent in $\left(R^{(i)}, m^{(i)}\right)$. Consequently, $S_{i}$ is a minimal basis for $m^{(i)}$. Thus each $R^{(i)}$ is an unramified complete regular noetherian local ring for the natural topology which has $k$ as a coefficient ring. By Theorem 15 of [5], each $u_{i}$ is bijective and so is $u^{\prime}: k^{\prime} \rightarrow R^{\prime}=U_{i \in N} R^{(i)}$. Let $k_{\omega}$ be equipped with the $m$-natural topology and $\left\{B_{r}\right\}$ the basis of neighborhoods of 0 which induces the topology, where $B_{r}$ is defined by (*) in $\S 1$. Regarding $R^{\prime}$ and $k^{\prime}$ as subspaces of $R$ and $k_{\omega}$ respectively, we assert that $u^{\prime}$ is a homeomorphism. Evidently $u^{\prime}$ is continuous by Proposition 2. For each $n \in N$ let us consider the complete noetherian local ring $\left(R^{(n)}, m^{(n)}\right)$ for the natural topology. By virtue of [12, p. 270, Th. 13], there must exist a $t \in N_{0}$ such that $R^{(n)} \cap q_{t} \subseteq m^{(n) n}$; hence $u_{n}^{-1}\left(R^{(n)} \cap q_{t}\right) \subseteq u_{n}^{-1}\left(m^{(n) n}\right)=\left(p, x_{1}, x_{2}, \cdots, x_{n}\right)^{n} k_{n} \subseteq k_{n} \cap B_{n}$. If $i>n$, then $u_{i}^{-1}\left(R^{(i)} \cap q_{t}\right) \subseteq k_{i} \cap B_{n}$ because $R^{(n)} \subset R^{(i)}$ and $u_{i}^{-1}\left(a_{j}\right)=$ $x_{j-1} \in k_{i} \cap B_{n}$ for all $j$ such that $i \geqq j>n$. It follows that $u^{\prime-1}$ $\left(R^{\prime} \cap q_{t}\right) \subseteq k^{\prime} \cap B_{n}$, Since $u^{\prime-1}\left(R^{\prime} \cap q_{t}\right)=U_{i \in N_{0}} u_{i}^{-1}\left(R^{(i)} \cap q_{t}\right)=U_{i \geqq n} u_{i}^{-1}$ $\left(R^{(i)} \cap q_{t}\right)$. Thus $u^{\prime-1}$ is continuous; therefore $u^{\prime}$ is a homeomorphism. Moreover, $R^{\prime}=u^{\prime}\left(k^{\prime}\right)$ is dense in $R$, for $k^{\prime}$ is dense in $k_{\omega}$ and $u$ is a surjective continuous homomorphism by Proposition 2 . We can conclude that $u$ is an isomorphism from [3, p. 41, Proposition 5]; therefore $R$ is a unique factorization domain by ]7]. (2) We also prove only for the case that $I=N$ and $k$ is a $v$-ring. The first half of the statement is evident from (2) of Theorem 3, where we take $D=(0)$. Since each element $c$ of $m=(p)$ can be written in the form that $c=\alpha q^{\lambda}$ for some unit $\alpha$ of $k$ and $\lambda \in N$, it is easy to see that $\operatorname{gr}\left(k_{\omega}\right)=$ $\bigoplus_{n \in N_{0}} B_{n} / B_{n+1}=k / m\left[p^{*}, x_{1}^{*}, x_{2}^{*}, \cdots\right]$. Hence $T=\{p\} U\left\{x_{i}\right\}_{i \in N}$ is a formal system of generators for $k_{\omega}$ by [4, p. 9, Proposition 1]. Let $E$ be an arbitrary finite subset of $T$, say $E=\left\{p, x_{1}, x_{2}, \cdots, x_{j}\right\}$. Let $\psi(p, x)=\sum_{i} f^{(i)} p^{\lambda_{i 0}} x_{1}^{\lambda_{i 1}} \cdots x_{j}^{\lambda_{i j}}$ be a form of degree $n$ in the elements of $E$ over $k_{\omega}$, nemely, $\lambda_{i 0}+\lambda_{i 1}+\cdots+\lambda_{i j}=n$ and $f^{(i)} \in k_{\omega}$, for each $i$ in the finite sum $\sum_{i}$. If $\psi(p, x)=0$, then $0=P_{j}(\psi(p, x))=$

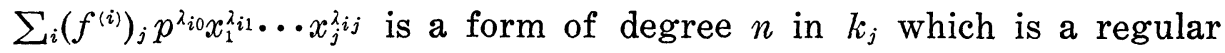
noetherian local ring with the maximal ideal generated by $E$. 
Hence, each $\left(f^{(i)}\right)_{j}$ is a nonunit of $k_{j}$, and so is $f^{(i)}$ in $k_{\omega}$. Thus the elements of $E$ are analytically independent in $k_{\omega}$. This completes the proof of the theorem.

\section{BIBLIOGRAPHY}

1. B. Ballet, Structure des anneaux strictement lineairement compacts commutatifs, C. R. Acad. Sci. Paris 266 (1968), 1113-1116.

2. N. Bourbaki, Topologie Générale, Chap. I-II, $3^{e}$ ed, Herman, Paris, 1961.

3. - Topologie Générale, Chap. III-IV, Herman, Paris, 1960.

4. — Algébre Commutative, Chap. III-IV, Herman, Paris, 1961.

5. I. S. Cohen, On the structure and ideal theory of complete local rings, Trans. Amer. Math. Soc. 59 (1946), 54-106.

6. B. Kolman, On a theorem in complete A-adic rings, Proc. Amer. Math. Soc. 19 (1968), 681-684.

7. C. P. Lu, On the unique factorization theorem in the ring of number theoretic functions, Illinois, J. Math. 9 (1965), 40-46.

8. M. Nagata, Local Rings, Interscience, 1962.

9. D. G. Northcott, Ideal theory, Cambridge Tracts, 421593.

10. P. Roquette, Abspaltung des Radikals in vollständingen lokalen Ringen, Hamburg Vniv. Math. Seminar Abhanlungen 21-23 (1958), 75-113.

11. S. Warner, Compact Noetherian rings, Math. Ann. 141 (1960), 161-170.

12. O. Zariski, and P. Samuel, Commutative Algebra, vol. II, Princeton, Van Nostrand, 1960.

Received September 23, 1969.

UNIVERSITY OF COLORADO 


\title{
PACIFIC JOURNAL OF MATHEMATICS
}

\author{
EDITORS
}

H. SAMELSON

Stanford University

Stanford, California 94305

C. R. Новву

University of Washington

Seattle, Washington 98105
J. DUGUNDJI

Department of Mathematics

University of Southern California

Los Angeles, California 90007

RICHARD ARENS

University of California

Los Angeles, California 90024

\section{ASSOCIATE EDITORS}

E. F. BECKENBACH

B. H. NeUManN

F. WOLF

K. YosHidA

\section{SUPPORTING INSTITUTIONS}

UNIVERSITY OF BRITISH COLUMBIA CALIFORNIA INSTITUTE OF TECHNOLOGY UNIVERSITY OF CALIFORNIA MONTANA STATE UNIVERSITY UNIVERSITY OF NEVADA NEW MEXICO STATE UNIVERSITY OREGON STATE UNIVERSITY UNIVERSITY OF OREGON OSAKA UNIVERSITY UNIVERSITY OF SOUTHERN CALIFORNIA
STANFORD UNIVERSITY UNIVERSITY OF TOKYO UNIVERSITY OF UTAH WASHINGTON STATE UNIVERSITY UNIVERSITY OF WASHINGTON

AMERICAN MATHEMATICAL SOCIETY CHEVRON RESEARCH CORPORATION TRW SYSTEMS

NAVAL WEAPONS CENTER 


\section{Pacific Journal of Mathematics}

\section{Vol. 36, No. $1 \quad$ November, 1971}

Norman Larrabee Alling, Analytic and harmonic obstruction on

nonorientable Klein surfaces ............................ 1

Shimshon A. Amitsur, Embeddings in matrix rings .............. 21

William Louis Armacost, The Frobenius reciprocity theorem and essentially bounded induced representations ....................... 31

Kenneth Paul Baclawski and Kenneth Kapp, Topisms and induced

non-associative systems ............................ 45

George M. Bergman, The index of a group in a semigroup ............ 55

Simeon M. Berman, Excursions above high levels for stationary Gaussian

processes....................................... 63

Peter Southcott Bullen, A criterion for $n$-convexity .............. 81

W. Homer Carlisle, III, Residual finiteness of finitely generated commutative

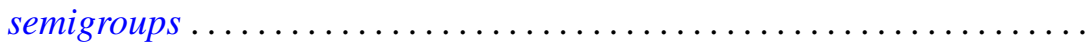

Roger Clement Crocker, On the sum of a prime and of two powers of

two ............................................ 103

David Eisenbud and Phillip Alan Griffith, The structure of serial rings . . . 109

Timothy V. Fossum, Characters and orthogonality in Frobenius

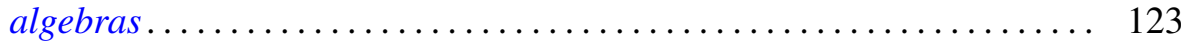

Hugh Gordon, Rings of functions determined by zero-sets . .......... 133

William Ray Hare, Jr. and John Willis Kenelly, Characterizations of Radon partitions...

Philip Hartman, On third order, nonlinear, singular boundary value

problems

David Michael Henry, Conditions for countable bases in spaces of

countable and point-countable type ..

James R. Holub, Hilbertian operators and reflexive tensor products ...

Robert P. Kaufman, Lacunary series and probability ..... . .

195

Erwin Kreyszig, On Bergman operators for partial differential equations in

two variables ................................

Chin-pi Lu, Local rings with noetherian filtrations . .

Louis Edward Narens, A nonstandard proof of the Jordan curve theorem...

S. P. Philipp, Victor Lenard Shapiro and William Hall Sills, The Abel summability of conjugate multiple Fourier-Stieltjes integrals. .

Joseph Earl Valentine and Stanley G. Wayment, Wilson angles in linear normed spaces

Hoyt D. Warner, Finite primes in simple algebras ...

Horst Günter Zimmer, An elementary proof of the Riemann hypothesis for an elliptic curve over a finite field... 